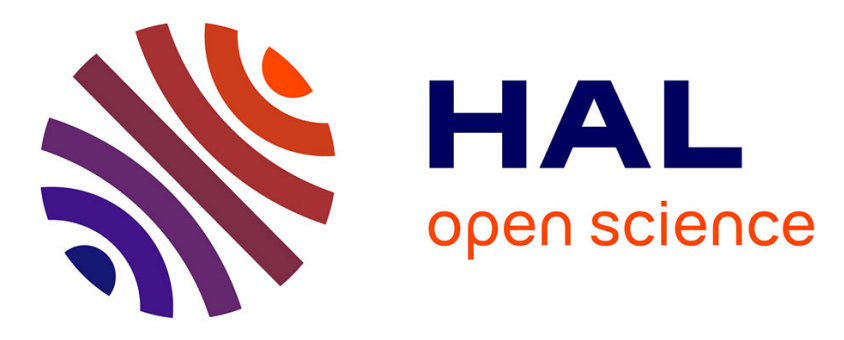

\title{
The thermally connected traps model applied to the thermoluminescence of Eu2+ doped Ba13-xAl22-2xSi10+2xO66 materials $(\mathrm{x} \sim 0.6)$.
} Grégoire Denis, Philippe Deniard, Xavier Rocquefelte, Mourad Benabdesselam, Stéphane Jobic

\section{To cite this version:}

Grégoire Denis, Philippe Deniard, Xavier Rocquefelte, Mourad Benabdesselam, Stéphane Jobic. The thermally connected traps model applied to the thermoluminescence of Eu2+ doped Ba13-xAl22-2xSi10+2xO66 materials $(\mathrm{x} \sim 0.6)$.. Optical Materials, 2010, 32 (9), pp.941. 10.1016/j.optmat.2010.01.029 . hal-00530703

\section{HAL Id: hal-00530703 https://hal.science/hal-00530703}

Submitted on 29 Oct 2010

HAL is a multi-disciplinary open access archive for the deposit and dissemination of scientific research documents, whether they are published or not. The documents may come from teaching and research institutions in France or abroad, or from public or private research centers.
L'archive ouverte pluridisciplinaire HAL, est destinée au dépôt et à la diffusion de documents scientifiques de niveau recherche, publiés ou non, émanant des établissements d'enseignement et de recherche français ou étrangers, des laboratoires publics ou privés. 


\title{
The thermally connected traps model applied to the thermoluminescence of $\mathrm{Eu}^{2+}$ doped $\mathrm{Ba}_{13-\mathrm{x}} \mathrm{Al}_{22-2 \mathrm{x}} \mathrm{Si}_{10+2 \mathrm{x}} \mathrm{O}_{66}$ materials $(\mathrm{x} \sim 0.6)$.
}

\author{
G. Denis ${ }^{1, *}$, P. Deniard ${ }^{1}$, X. Rocquefelte ${ }^{1}$, M. Benabdesselam ${ }^{2}$ and S. Jobic ${ }^{1}$ \\ ${ }^{1}$ Institut des Matériaux Jean Rouxel (IMN), Université de Nantes, CNRS, 2 rue de la Houssinière, BP \\ 32229, 44322 Nantes cedex 3, France \\ ${ }^{2}$ Université de Nice-Sophia Antipolis, Laboratoire de Physique de la Matière Condensée, CNRS UMR \\ 6622, Parc Valrose, 06108 Nice Cedex 2, France
}

* Corresponding author.

Institut des Matériaux Jean Rouxel (IMN), Université de Nantes, CNRS, 2 rue de la Houssinière, BP 32229, 44322 Nantes cedex 3, France, tel.33240373938, gdenis.professional@gmail.com

\begin{abstract}
The thermoluminescence (TL) properties of the $\mathrm{Eu}^{2+}$ doped $\mathrm{Ba}_{13-\mathrm{x}} \mathrm{Al}_{22-2 \mathrm{x}} \mathrm{Si}_{10+2 \mathrm{x}} \mathrm{O}_{66} \quad(\mathrm{x} \sim 0.6)$ phosphorescent materials (hereafter labeled BASO:Eu) are analyzed on the basis of the thermally connected traps (TCT) model. Basically, in these materials, $\mathrm{Eu}^{2+}$ cations are photo-ionized under UV excitation, and electrons are trapped at defects. The return to the ground state is thermo-stimulated, and the freed electrons recombine at photo-oxidized activator sites with emission of light. TL properties between 80 and $650 \mathrm{~K}$, as well as an isothermal emission decay (IED) at room temperature, were collected for BASO:Eu1\% and BASO:Eu0.5\% samples to characterize the phosphorescence mechanism in this series of materials and to determine the trap depths. Thermoluminescence glow curves of BASO:Eu excited either at $254 \mathrm{~nm}$ or at $365 \mathrm{~nm}$, or excited at 254 $\mathrm{nm}$ for durations of $10 \mathrm{~s}$ and $240 \mathrm{~s}$, as well as the IED, agree well with a TCT model. Based on our analysis, phosphorescence in BASO:Eu compounds is associated with the depletion of five traps with depths of $0.56,0.61$, $0.65,0.69$ and $0.73 \mathrm{eV}$.
\end{abstract}

Keywords. Thermoluminescence, phosphorescence, trap, europium, aluminosilicate PACS. 78.60.K, 78.55, 72.20.J

\section{Introduction}


Phosphorescence, also named persistent luminescence or afterglow, refers to an emission of light at room temperature (RT) that persists once the UV-vis excitation is turned off. This phenomenon, which occurs in wide band gap materials exclusively, arises from the trapping of electrons and/or holes at defects (namely, impurities, anionic or cationic vacancies...) under the excitation of the phosphor. After removal of the illumination source, the absorbed energy is progressively released, that is the photo-generated charge carriers are thermally detrapped before their recombination at the luminescent centers. This explains the delayed light emission. Consequently, phosphorescence may be viewed as a thermoluminescence phenomenon occurring at RT.

Historically, the first manufactured phosphorescent inorganic material was $\mathrm{ZnS}: \mathrm{Cu}, \mathrm{Co}$ [1]. Commonly, this compound was co-doped with tritium to enhance its phosphorescence duration, the field of application being then significantly restricted due to the presence of this radioactive element. In 1995, Matsuzawa et al. reported on the outstanding long persistent luminescence of $\mathrm{SrAl}_{2} \mathrm{O}_{4}: \mathrm{Eu}^{2+}$, $\mathrm{Dy}^{3+}$ which can last several hours without addition of any radioelement [2]. Since this date, numerous investigations have been dedicated to europium activated aluminates, silicates and alumino-silicates and their co-doped derivatives [3-7]. In this framework, we recently reported on the phosphorescent properties of new $\mathrm{Eu}^{2+}$ doped $\mathrm{Ba}_{13-\mathrm{x}} \mathrm{Al}_{22-2 \mathrm{x}} \mathrm{Si}_{10+2 \mathrm{x}} \mathrm{O}_{66}$ compounds (x 0.6), hereafter labeled BASO:Eu [8]. These phases exhibit a highly distorted tridymite structure with three distinct crystallographic sites for barium. Under UV excitation, the $\mathrm{Eu}^{2+}$ activators localised at the $\mathrm{Ba} 1, \mathrm{Ba} 2$ and $\mathrm{Ba} 3$ sites lead to three emission bands located at $2.96 \mathrm{eV}(419 \mathrm{~nm}), 2.81 \mathrm{eV}(442 \mathrm{~nm})$, and $2.26 \mathrm{eV}(549 \mathrm{~nm})$, respectively. The overall emission extends on the whole visible spectrum which explains the white fluorescence perceived with naked eyes under UV illumination. After stopping the illumination, a bluish-white afterglow is perceptible for several minutes.

In order to achieve the characterization of the BASO:Eu long persistent phosphors thermoluminescence (TL) measurements coupled to room temperature isothermal emission decay (RT-IED) collections were carried out. Results are reported here and discussed on the basis of the thermally connected traps (TCT) model.

\section{Experimental}


Synthesis. $\mathrm{Ba}_{13-\mathrm{x}} \mathrm{Al}_{22-2 \mathrm{x}} \mathrm{Si}_{10+2 \mathrm{x}} \mathrm{O}_{66}: \mathrm{Eu}$ polycrystalline samples were prepared through solid-statereactions. Barium carbonate $\left(\mathrm{BaCO}_{3} 99.997 \%\right.$ Alfa Aesar), alumina $\left(\mathrm{Al}_{2} \mathrm{O}_{3} 99.997 \%\right.$ Alfa Aesar), silica $\left(\mathrm{SiO}_{2} 99.99 \%\right.$ Chempur), and europium oxide $\left(\mathrm{Eu}_{2} \mathrm{O}_{3} 99.99 \%\right.$ Alfa Aesar) were weighted with a slight excess of silicon compared to the aimed $\mathrm{Ba}_{13} \mathrm{Al}_{22} \mathrm{Si}_{10} \mathrm{O}_{66}$ chemical composition [8]. The blend was ball milled and heated at $1350^{\circ} \mathrm{C}$ under reducing argon/hydrogen atmosphere. Samples underwent a second firing in similar conditions after an intermediate grinding step. The purity of the materials was systematically checked by an X-ray structural refinement thanks to a Rietveld procedure [9]. A full structural characterization of the materials is reported in [8].

Thermoluminescence measurements. $4 \mathrm{mg}$ of the sample was dispersed in acetone and was settled on an aluminium cell by sedimentation technique. TL readout was performed from 80 to $600 \mathrm{~K}$ or from 300 to $650 \mathrm{~K}$ with a linear heating rate of $1 \mathrm{~K} / \mathrm{s}$ under high vacuum $\left(10^{-5} \mathrm{Torr}\right)$. Prior to any TL acquisition, the samples were brought up to $650 \mathrm{~K}$ in the dark to definitely empty traps and then cooled down at liquid nitrogen temperature or room temperature. The UV illumination was made in situ with a low-pressure UV mercury lamp working either at 254 or at $365 \mathrm{~nm}$ (Vilber Lourmat) for periods of $10 \mathrm{~s}$ or $240 \mathrm{~s}$. Samples were placed at $15 \mathrm{~cm}$ from the lamps. The excitation power density was estimated to be 340 and $400 \mathrm{~mW} . \mathrm{cm}^{-2}$ for the $254 \mathrm{~nm}$ and $365 \mathrm{~nm}$ lamps, respectively. After a $60 \mathrm{~s}$ delay, the temperature was raised and the TL signal was recorded by means of a S13 response photomultiplier (integration of emission over a 250 - $600 \mathrm{~nm}$ interval; XP-2018B, Philips).

Isothermal emission decays (IED). The phosphorescence decay of BASO:Eu (reported in [8]) was recorded after a $5 \mathrm{mn}$ excitation (324 nm emission of a Xenon lamp) into a Fluorolog-3 spectrophotometer (Jobin Yvon). The emission decay fits were carried out with the Origin 7.5 software (OriginLab Corporation USA).

\section{Detrapping process in BASO:Eu}

\subsection{Thermoluminescence}

The TL glow curve of BASO:1\%Eu irradiated at $80 \mathrm{~K}$ for 240 s is displayed in figure 1 . The TL signal, integrated onto the UV-visible region of the electromagnetic spectrum, extends on the whole probed temperature domain, i.e. from 80 to $600 \mathrm{~K}$. Especially, the TL glow curve is composed of a 
large band lying from 80 to $220 \mathrm{~K}$ with a maximum at $171 \mathrm{~K}$ and a shoulder at $181 \mathrm{~K}$, and a bundle of peaks ranging from 220 to $600 \mathrm{~K}$ with maxima and shoulders at 236, 247, 263, 292, 314, 330, and 348 K. This clearly evidences the existence in the material of several traps with well defined depths. Beyond $350 \mathrm{~K}$, a slow decrease of the TL intensity is observed and no peak is observed. This slow decrease might be correlated to thermal quenching of $\mathrm{Eu}^{2+}$ luminescence that begins around $370 \mathrm{~K}$ in BASO:Eu [10]. Based on this experiment, at least nine distinguishable traps are detected in BASO:Eu compounds.

The TL glow curve of BASO:1\%Eu irradiated at room temperature for $240 \mathrm{~s}$ at $\lambda_{\text {exc }}=254 \mathrm{~nm}$ is given in figure 2. The TL signal is then only composed of a very broad band spreading from 293 to $650 \mathrm{~K}$ with a TL intensity maximum at $340 \mathrm{~K}$ (width at half maximum of about $100 \mathrm{~K}$ ). It is worth noticing that the TL peaks at 292, 314, 330, and $348 \mathrm{~K}$ observed after a read out from nitrogen temperature (figure 1) are not detected at all here. This might originate from a concomitant release of the electrons associated with these traps at room temperature (see below).

Before discussing deeper the TL measurements, it is instructive to consider briefly the predictable phosphorescence mechanism at work in BASO:Eu. As for $\mathrm{Eu}^{2+}$ doped aluminates, such as $\mathrm{SrAl}_{2} \mathrm{O}_{4}: \mathrm{Eu}, \mathrm{Dy}, \mathrm{CaAl}_{2} \mathrm{O}_{4}: \mathrm{Eu}, \mathrm{Nd}$, and $\mathrm{Sr}_{4} \mathrm{Al}_{14} \mathrm{O}_{25}: \mathrm{Eu}$,Dy [11-15], we may expect that photoionization of $\mathrm{Eu}^{2+}$ cations takes place in BASO:Eu under UV-excitation. This photoionization leads to the creation of a metastable state formed of $\mathrm{Eu}^{3+}$ cations and freed electrons trapped at defects. The backwards reaction occurs when the excitation source is turned off and consists in a thermal detrapping of the electron at room temperature with radiative recombination at the photo-ionized activator sites. First, preliminary photoconductivity measurements carried out on BASO:Eu compounds revealed the appearance of a photo-current under UV illumination. This suggests that photogenerated electron migrate towards defects via the conduction band (CB). Second, the charge transfer $\mathrm{O}^{2-} \rightarrow \mathrm{Eu}^{3+}$ in oxidized BASO:Eu compounds is measured around $4.70 \mathrm{eV}$ [8], which locates the ${ }^{8} \mathrm{~S}_{7 / 2}$ ground state of $\mathrm{Eu}^{2+}$ at $4.70 \pm 0.50 \mathrm{eV}$ above the valence band (VB) according to the Dorenbos model [15]. In addition, the zero phonon line of $4 \mathrm{f}^{6} 5 \mathrm{~d}^{1} \rightarrow 4 \mathrm{f}^{7} \mathrm{Eu}^{2+}$ transition can be estimated at about $3 \mathrm{eV}[10]$. This locates the lowest $4 \mathrm{f}^{6} 5 \mathrm{~d}^{1}$ state around $7.70 \pm 0.50 \mathrm{eV}$ above the VB, 
that is likely a few hundreds of meV below the $\mathrm{CB}$, as suggested by the presence of the photocurrent under UV excitation. Band gap measurement of the BASO host by UV-vis spectroscopy would require an UV-vacuum set up and could not be realized at the laboratory. Based on the aforementioned arguments, a schematic proposal of the energy levels in BASO:Eu is depicted in figure 3.

The excited states of $\mathrm{Eu}^{2+}$ are then probably positioned nearby the $\mathrm{CB}$ which makes easy the photo-ionization of $\mathrm{Eu}^{2+}$ activators under UV excitation. This conclusion can also readily be derived from the TL glow curves of BASO:0.5\% Eu excited at 365 and $254 \mathrm{~nm}$ (figure 4). Actually, the TL intensity is reduced by one order of magnitude when the excitation wavelength increases from $254 \mathrm{~nm}$ $(4.88 \mathrm{eV})$ to $365 \mathrm{~nm}(3.40 \mathrm{eV})$. This suggests that the higher the excitation energy, the higher the probability to photo-ionize activators (due to the more pronounced delocalized character of the excited levels), and the higher the concentration of filled electron traps $(f)$ just after excitation.

Let us now examine the figure 4 more carefully. The BASO:0.5\% Eu TL glow curve can arbitrary be decomposed into three temperature intervals, that is i) the $\mathrm{S}$ area, ranging from 80 to $175 \mathrm{~K}$, associated with shallow traps, ii) the I area, from 175 to $300 \mathrm{~K}$, associated with intermediate depth traps, and iii) the $\mathrm{H}$ area (above $300 \mathrm{~K}$ ) associated with high depth traps. The TL intensities integrated in these temperature intervals are labeled $I_{S}, I_{I}$ and, $I_{H}$, respectively. All $I_{H} / I_{S}, I_{H} / I_{I}$, and $I_{I} / I_{S}$ intensity ratios increase when illumination wavelength shifts from 254 to $365 \mathrm{~nm}$ (table 1). In other words, there is an enhanced TL intensity transfer from low temperature peaks towards high temperature peaks when $f$ decreases. Such a TL intensity transfer was already reported into $\mathrm{ZnS}: \mathrm{Cu}$ compounds and was assigned to the occurrence of a thermal connection between the traps [16]. Namely, when temperature is raised, a significant portion of the electrons released from $\mathrm{S}$ traps may be captured by both I and $\mathrm{H}$ traps, while electrons freed from I traps may be only captured by the H traps. In the framework of such a thermally connected traps model (TCT model), the portion of transferred electrons grows when the concentration of free traps increase, i.e. when $f$ decreases [17,18]. In consequence, both $I_{H} / I_{S}, I_{H} / I_{I}$, and $I_{I} I_{S}$ intensity ratios should increase when illumination wavelength shifts from 254 to $365 \mathrm{~nm}$. The calculated ratios reported in table 1 follow this statement.

Of course, the $f$ parameter can also be tuned by modifying the UV-excitation duration. The TL 
glow curves of BASO:0.5\%Eu sample irradiated during $10 \mathrm{~s}$ and $240 \mathrm{~s}$ are depicted in figure 5 . The TL peaks after the $240 \mathrm{~s}$ illumination is one order of magnitude more intense than those after a $10 \mathrm{~s}$ excitation. According to the TCT model, the decreasing of the total intensity when the illumination period decreases from $240 \mathrm{~s}$ to $10 \mathrm{~s}$ should lead to an enhancement of the intensity transfer from shallower to deeper traps. Again, this intensity transfer is asserted on the basis of the calculation of the $I_{H} / I_{S}, I_{H} / I_{I}$, and $I_{I} / I_{S}$ ratios given in table 2 since these latter increase when the excitation duration decreases.

\subsection{Isothermal emission decay and TL correlation}

TL is usually considered as the powerful and appropriate characterization method for phosphorescent materials, but RT-IED acquisition may be also of a great interest. Since this latter is straightforward, this has been largely employed for phosphorescent materials characterization, in particular for a classification of the phosphors in terms of time emission. For instance, the isothermal afterglow of $\mathrm{Eu}^{2+}$ has been fitted in different hosts (e.g. $\left.\mathrm{SrSiO}_{3}, \mathrm{SrAl}_{2} \mathrm{O}_{4}, \mathrm{BaAl}_{2} \mathrm{O}_{4}\right)$ with multiple exponential decays [19-23]. In a similar way, we evidenced in reference [8] that the RT-IED of BASO: $1 \% \mathrm{Eu}^{2+}$ collected up to $900 \mathrm{~s}$ could be decomposed into five exponential functions. Such a decomposition may have two different origins. On one hand, several exponential curves may be always used, for practical reasons only and without physical significance, to fit an emission decay whatever the kinetics order of the detrapping process. On the other hand, each exponential function may correspond to the physical read out of a trap to which is associated a first order kinetics TL process [24].

Considering this latter case, from the RT-IED refined parameters, i.e. the starting intensity $I_{0}$ and the lifetime $\tau$ of the trapped state extracted from the exponential decay law $I(t)=I_{0} \exp (-t / \tau)$, can be estimated the trap depth $\left(E_{i}\right)$ and the number of trapped electrons at $\mathrm{t}=0\left(n_{0 i}\right)$ of traps $i$ via the formulae $E_{i}=k T \ln \left(\tau_{i} s\right.$ ) and $n_{0 i}=I_{0 i} \tau_{i}$ (where $s$ is the detrapping frequency factor and $k$ the Boltzmann constant) [25]. Based on RT-IED refinement data reported in table 4 of reference [8], this protocol leads to the calculated parameters $n_{0 i}$ and $E_{i}$ listed in table 3 for pre-exponential factor $s$ ranging from $10^{9}$ to $10^{13} \mathrm{~s}^{-1}$. As expected, the deeper the trap depth $E_{i}$, the higher the trapped electrons 
concentration $n_{0 i}$. Moreover, it can be observed that an increasing of the frequency factor by one order of magnitude results in a $\sim 0.06 \mathrm{eV}$ increase of the trap depth.

Once $n_{0 i}$ and $E_{i}$ parameters are known, the TL signal associated with each trap may be simulated on the basis of a first order kinetics detrapping-recombination process according to equation 1:

$$
I_{T L}(T)=n_{0} s e^{\left(-\frac{E}{k T}\right)} \exp \left[-\frac{s}{q} \int_{T_{0}}^{T} e^{\left(-\frac{E}{k T_{1}}\right)} d T_{1}\right]
$$

where $q$ and $T_{0}$ are the heating rate and the starting temperature, respectively [25]. The simulation of the TL signals associated with the five traps detected from RT-IED is depicted in figure 6 for $s$ equal to $10^{10} \mathrm{~s}^{-1}$. The simulated curves evidence TL peaks maxima at $270,293,309,327$, and $344 \mathrm{~K}$, that are at temperatures very similar to those determined experimentally $(263,292,314,330$ and $348 \mathrm{~K}$, respectively (figure 1)). This suggests that the persistent phosphorescence (i.e. RT-IED) of BASO: $1 \% \mathrm{Eu}$ can be attributed, at least during the first $900 \mathrm{~s}$ of the luminescence decay, to the read out of these five specific traps only. For the sake of accuracy, the best goodness of fit related to the positioning in temperature of the simulated and experimental TL peaks maxima is obtained for detrapping frequency $s$ ranging from $10^{9}$ to $10^{10} \mathrm{~s}^{-1}$. This corresponds to trap depths nearby $0.56,0.61$, $0.65,0.69$ and $0.73 \mathrm{eV}$ with a systematic error of $0.03 \mathrm{eV}$ according to table 3 . At this point, let us emphasize the fact that figure 7 is not a tentative refinement of figure 1, but a simulation of the overall TL curve on the basis of external parameters extracted from a RT-IED analysis. Consequently, it turns out to be natural that the relative intensity ratios between peaks are different for the two figures, the general features of a TL spectrum being strongly dependent on the history of the material and the electron population $n_{0 i}$ at each defect trap after excitation in particular. A major point to stress on here is the feasibility to detect the existence of five distinct traps with a rough refinement of the RT-IED. This may underline that the RT-IED refinement with multiple exponential decays is reliable and consequently that the isothermal detrapping-recombination mechanism of the five considered traps may be close to a first order kinetics process. Here again, this behavior could be related to a thermal connection of the traps. Indeed, TL simulations performed in the framework of the TCT model demonstrated that the presence of deep traps involved the appearance of a fake first order kinetic process for the detrapping of the low depth traps [18]. In the case of BASO:Eu, we may envisage that 
the existence of deep traps asserted by the TL signal beyond $450 \mathrm{~K}$ leads to a fake first order detrapping process of traps associated with 263, 292, 314, 330 and 348 K TL peaks. Furthermore, it is worthwhile to note that the RT-IED measurements reveal the influence of the $263 \mathrm{~K}$ TL peak on the detrapping mechanism at room temperature. This conducts us to conclude that the trap associated with this specific TL peak is partially occupied under excitation at $293 \mathrm{~K}$.

Let us now compare the TL signal simulated with the estimated traps depth to the experimentally measured TL glow curve after the excitation at room temperature (Figure 2). In order to achieve such a comparison, it is essential to simulate the TL peaks by taking into account the $60 \mathrm{~s}$ time imposed between the end of the illumination and the beginning of the TL acquisition (see section 2). During this idle time, the five aforementioned traps undergo electron depletion at room temperature. As a consequence, the real trapped electron concentrations to consider at the beginning of the TL measurement, labeled $n_{i 60}$, corresponds to the intensity measured $60 \mathrm{~s}$ after the cessation of the UV excitation during the IED acquisition, labeled $I_{i 60}$. The $n_{i 60}$ populations, calculated from the IED refinement results via the expression $n_{i 60}=I_{i 60} \tau_{i}$, are then equal to $3 \times 10^{0}, 6.8 \times 10^{4}, 1.8 \times 10^{6}, 6.5 \times 10^{6}$ and $1.84 \times 10^{7}$ a.u. for traps 1 to 5 of BASO:1\%Eu, respectively. The TL glow curve simulated with these populations is displayed in figure 7 and compared to experimental curve of figure 2 . The rising of the measured TL intensity is well fitted by the simulated TL curve but not the high temperature part. The lack of agreement between simulated and experimental curves above $350 \mathrm{~K}$ can be partially explained. Indeed, the TL signal at high temperature can be attributed to deep traps that does not give rise to distinct $\mathrm{TL}$ peaks because of the $\mathrm{Eu}^{2+}$ thermal quenching above $370 \mathrm{~K}$. These deep traps are not released during the $900 \mathrm{~s}$ IED acquisition at RT and are then not taken into account in the simulation. As a result, the lack of agreement above $450 \mathrm{~K}$ can be considered as a logical consequence of the employed procedure in regard of the intrinsic properties of the probed material. Thus, all the attention must be paid to the low temperature part of the TL curves only. At these temperatures, the rising of the measured TL intensity is well fitted by the simulated TL curve. This may evidence the validity of the estimated traps parameters and, implicitly, a detrapping process via a (fake) first order kinetics in agreement with the TCT model. 


\section{Conclusion}

From thermoluminescence measurements, it appears that BASO:Eu compounds contain several traps with some of them located in the temperature range that can induce phosphorescence. Indeed, at least nine well-defined peaks can be identified from the analysis of the TL glow curve collected in the 80-650 K temperature range. More especially, on the basis of the decomposition of the RT isothermal emission decay by means of a sum of exponential functions followed by the satisfying reconstruction of the TL glow curves, the long persistence luminescence of BASO:Eu at RT may be truly attributed to the depletion of five traps in particular. These later are characterized by a depth of $0.56(3), 0.61(3)$, $0.65(3), 0.69(3)$ and $0.73(3) \mathrm{eV}$, with a detrapping frequency factor $(s)$ of about $10^{9}-10^{10} \mathrm{~s}^{-1}$, and are at the origin of the TL peaks at 263, 292, 314, 330 and $348 \mathrm{~K}$, respectively. The apparent first order kinetics of their associated detrapping process suggests an overall mechanism related to a thermally connected traps model. This has been asserted via the modulation of the initially occupied traps fraction $f$ by a change in excitation wavelength and illumination time which strongly influences the relative intensities of the TL peaks. Namely, the lower the $f$ values, the higher the propensity of released electrons to be captured by deeper traps before recombination, and the higher the possibility of TL intensity transfer from the low temperature peak towards the high temperature peak. The aptness of a TCT model requires the existence of deep trap(s). The TL tail above $400 \mathrm{~K}$ asserts the presence of deep traps in BASO:Eu samples. A remaining point to address concerns the low value of the estimated detrapping frequency factor $\left(s\right.$ of $10^{9}-10^{10} \mathrm{~s}^{-1}$ ), significantly different from the expected value for oxides $\left(10^{13}-10^{14} \mathrm{~s}^{-1}\right)$. If it is not uncommon to find very low $s$ factors $\left(s=10^{8} \mathrm{~s}^{-1}\right.$ in $\left.\mathrm{LiLnSiO}_{4}: \mathrm{Ce}^{3+}, \mathrm{Sm}^{3+}[26]\right)$, such values are generally attributed to thermally activated tunneling detrapping [27]. This could suggest that a thermally connected trap model via a thermally assisted detrapping tunneling might occur also into BASO:Eu compounds. Extra theoretical work, experimental investigations as IED measurements at different temperatures and thermally stimulated conductivity measurements would be necessary to rise up this ambiguity. 


\section{References}

$1 \quad$ S. Shionoya, H. P. Kallmann, B. Kramer, Phys. Rev. 121 (1961) 1619

2 T. Matsuzawa, T. Aoki, N. Takeuchi, Y. Murayama, J. Electrochem. Soc. 143 (1996) 2670

3 F. Clabau, X. Rocquefelte, S. Jobic, P. Deniard, M. H. Whangbo, A. Garcia, T. LeMercier, Chem. Mater. 17 (2005) 3904

$4 \quad$ B. Liu, C. Shi, Z. Qi, App. Phys. Lett. 86 (2005) 191111

5 T. Aitasalo, J. Hölsa, J. Högne, M. Lastusaari, J. Niittykoski, J. Phy. Chem. B 110 (2006) 4589

$6 \quad$ D. Jia, W. Jia, Y. Jia, J. Appl. Phys. 101 (2007) 023520

7 H. Kubo, H. Aizawa, T. Katsumata, S. Komuro, T. Morikawa, J. Cryst. Growth 275 (2005) e1767-e1771

8 G. Denis, P. Deniard, E. Gautron, F. Clabau, A. Garcia, S. Jobic, Inorg. Chem. 47 (2008) 4226

9 V. Petricek, M. Dusek, L. Palatinus, The Crystallographic Computing System JANA 2006 Beta (2006) Praha, Czeck Republic: Academy of Sciences

10 G. Denis, Ph.D thesis (2008) University of Nantes, France

11 H. Yamamoto, T. Matsuzawa, J. Lumin. 72 (1997) 287

12 Y. Lin, Z. Tang, Z. Zhang, Materials Letters 51 (2001) 14

13 F. Clabau, X. Rocquefelte, S. Jobic, P. Deniard, M-H. Whangbo, A. Garcia, T. Le Mercier Solid State Science 9 (2007) 608-612

14 F. Clabau, X. Rocquefelte, T. Le Mercier, P. Deniard, S. Jobic, M. Whangbo

Chem. Mater, 18 (2006) 3212-3220

15 P. Dorenbos, J. Lumin. 111 (2005) 89

16 S. Shionoya, H. Kallmann, P. Hartmut, B. Kramer, Phys. Rev. 121 (1961) 1607-1619

17 T. Sakurai, R. K. Gartia, J. Phys. D: Appl. Phys. 36 (2003) 2719

18 S. Basun, G. F. Imbusch, D. D. Jia, W. M. Yen, J. Lumin. 104 (2003) 283

19 G. F. J. Garlick, A. F. Gibson, Proceedings of the physical society 60 (1948) 574

20 R. Sakai, T. Katsumata, S. Komuro, T. Morikawa, J. Lumin. 85 (1999) 149

21 V. Abbruscato, J. Electrochem. Soc. 118 (1971) 930

22 T. Katsumata, T. Nabae, K. Sasajima, S. Komuro, T. Morikawa, J. Electrochem. Soc. 144 (1997) L243

23 A. Nag, T. R. N. Kutty, Journal of Alloys and Compounds 354 (2003) 221

24 J. T. Randall, M. H. F. Wilkins, Proceedings of the Royal Society of London. Series A, Mathematical and Physical Sciences 184 (1945) 390

25 S. W. S. McKeever, Thermoluminescence of solids (2005) Cambridge: Cambridge university press.

26 A.V. Sidorenko, P. Dorenbos, A.J.J. Bos, C.W.E. van Eijk, P.A. Rodnyi, J. Phys. : Cond. Matt.18 (2006) 4503-4514

27 A. Vedda, M. Martini, F. Meinardi, J. Chval, M. Dusek,J. A. Mares, E. Mihokova, M. Nikl, Phys. Rev. B 61 (2000) 8081 


\section{Figures captions}

Figure 1. TL glow curve of BASO:1\%Eu irradiated with UV-254 nm for $240 \mathrm{~s}$ at $80 \mathrm{~K}$.

Figure 2. TL glow curve of BASO:1\%Eu irradiated with UV-254 nm for $240 \mathrm{~s}$ at $293 \mathrm{~K}$.

Figure 3. Energy band scheme of BASO:Eu

Figure 4. TL glow curves of BASO:0.5\%Eu excited at $254 \mathrm{~nm}$ ( - left scale) and $365 \mathrm{~nm}(\triangle-$ right scale) - (340 and $400 \mathrm{~mW} \cdot \mathrm{cm}^{-2}$ respectively, $\left.240 \mathrm{~s}, q=1 \mathrm{~K} \cdot \mathrm{s}^{-1}\right)$. The hatched areas correspond to the integrated intensities $I_{S}$ (see text).

Figure 5. TL glow curves of BASO:0.5\%Eu sample obtained after $10 \mathrm{~s} \mathrm{(o-} \mathrm{right} \mathrm{scale} \mathrm{)} \mathrm{and} 240 \mathrm{~s}$ (- left scale) $254 \mathrm{~nm}$ UV excitation at $80 \mathrm{~K}$.

Figure 6. TL glow curves simulated via equation 1 parameters issued from table $3\left(s=10^{10} \mathrm{~s}^{-1}\right)$.

Figure 7. Simulated and observed TL glow curve of BASO: $1 \%$ Eu excited at room temperature. The solid line is the sum of the five TL glow curves simulated according to equation 1 with $n_{0 i}$ equal to $3 \times 10^{0}, 6.8 \times 10^{4}, 1.8 \times 10^{6}, 6.5 \times 10^{6}$ and $1.84 \times 10^{7}$ and $s=10^{10} \mathrm{~s}^{-1}$. The two TL curves have been normalized to their maximum.

\section{Tables}

Table 1. Relative intensities ratio from BASO:Eu0.50\% TL glow curves after 254 and $365 \mathrm{~nm}$ excitation (240 s). $I_{S}, I_{I}$, and $I_{H}$ correspond to the TL peak intensities associated with traps cleaning within the $\mathrm{S}(80-175 \mathrm{~K}), \mathrm{I}(175-300 \mathrm{~K})$, and $\mathrm{H}$ (above $300 \mathrm{~K})$ temperature intervals.

\begin{tabular}{ccc} 
& $\lambda_{\text {Exc }}=254 \mathrm{~nm}$ & $\lambda_{\text {Exc }}=365 \mathrm{~nm}$ \\
\hline$I_{H} / I_{S}$ & $2.33 \pm 0.10$ & $8.33 \pm 0.70$ \\
$I_{H} / I_{I}$ & $0.80 \pm 0.10$ & $1.47 \pm 0.30$ \\
$I_{I} / I_{S}$ & $2.94 \pm 0.35$ & $5.56 \pm 1.00$ \\
\hline
\end{tabular}

Table 2. Relative intensities ratio from BASO:Eu0.50\% TL glow curves excited during 240 and $10 \mathrm{~s}$ at $254 \mathrm{~nm}$.

\begin{tabular}{ccc}
\hline & $240 \mathrm{~s}$ & $10 \mathrm{~s}$ \\
\hline$I_{H} / I_{S}$ & $2.33 \pm 0.10$ & $5.55 \pm 0.30$ \\
$I_{H} / I_{I}$ & $0.80 \pm 0.10$ & $1.61 \pm 0.25$ \\
$I_{I} / I_{S}$ & $2.94 \pm 0.35$ & $3.33 \pm 0.50$ \\
\hline \hline
\end{tabular}

Table 3. Initial trapped electron concentration $n_{0 i}$ and trap depth $E_{i}(i=1$ to 5) calculated from BASO:Eu1\% IED refinement (438 nm emission, $293 \mathrm{~K}, 900 \mathrm{~s}$ [8]) for different $s$ values.

\begin{tabular}{|c|c|c|c|c|c|c|c|}
\hline \multirow{2}{*}{$\begin{array}{c}\text { Trap } \\
I\end{array}$} & \multirow{2}{*}{$n_{0 i}$ (a.u.) } & \multicolumn{6}{|c|}{$E_{i}(\mathrm{eV})$} \\
\hline & & $s\left(\mathrm{~s}^{-1}\right)$ & $10^{9}$ & $10^{10}$ & $10^{11}$ & $10^{12}$ & $10^{13}$ \\
\hline 1 & $8.4510^{5}$ & & 0.53 & 0.59 & 0.64 & 0.70 & 0.76 \\
\hline 2 & $4.3710^{6}$ & & 0.58 & 0.64 & 0.69 & 0.75 & 0.81 \\
\hline 3 & $1.0010^{7}$ & & 0.62 & 0.68 & 0.74 & 0.80 & 0.86 \\
\hline 4 & $1.1910^{7}$ & & 0.66 & 0.72 & 0.78 & 0.84 & 0.90 \\
\hline 5 & $2.5010^{7}$ & & 0.70 & 0.76 & 0.82 & 0.88 & 0.94 \\
\hline
\end{tabular}




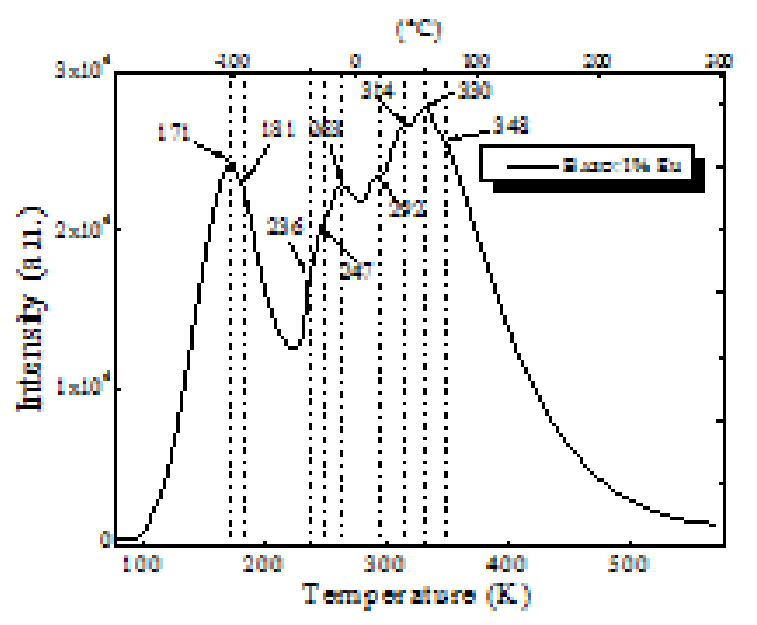

Figure 1

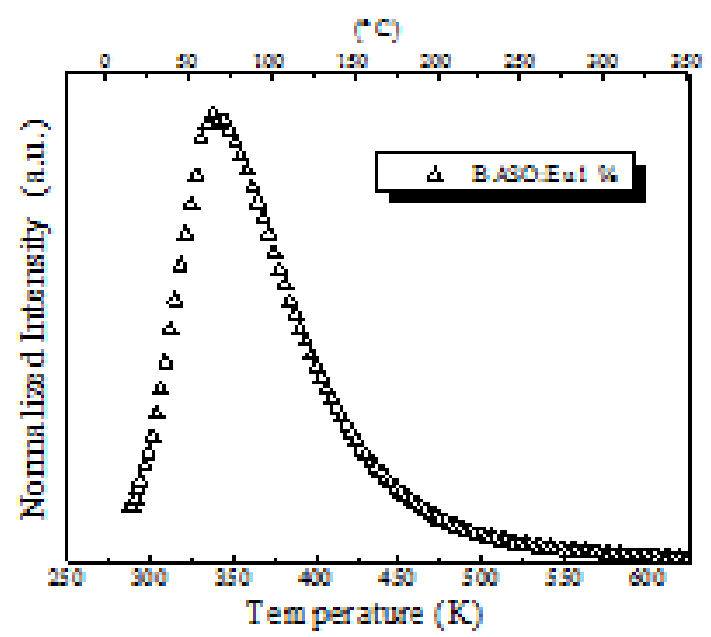

Figure 2

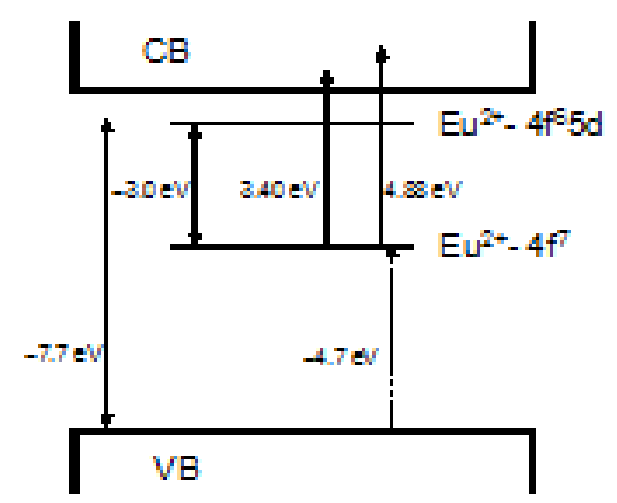

Figure 3 


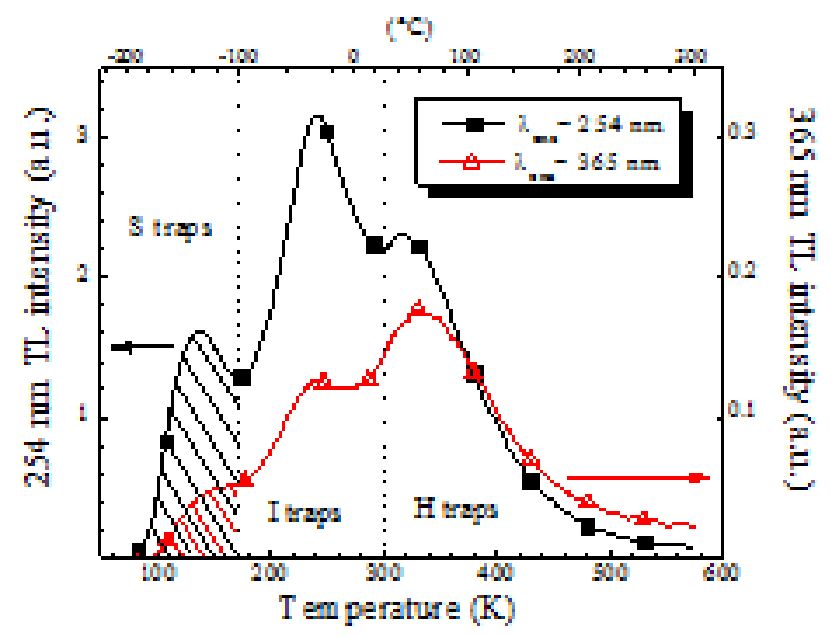

Figure 4

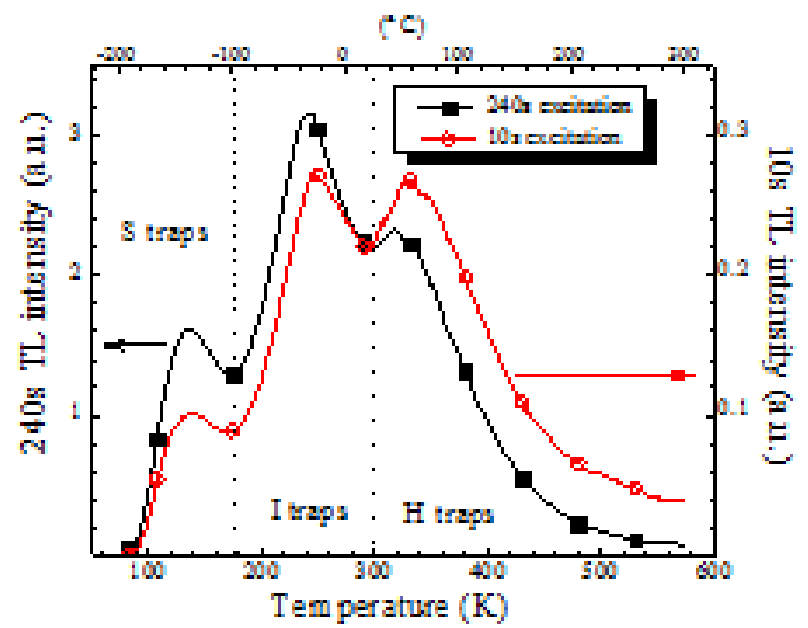

Figure 5

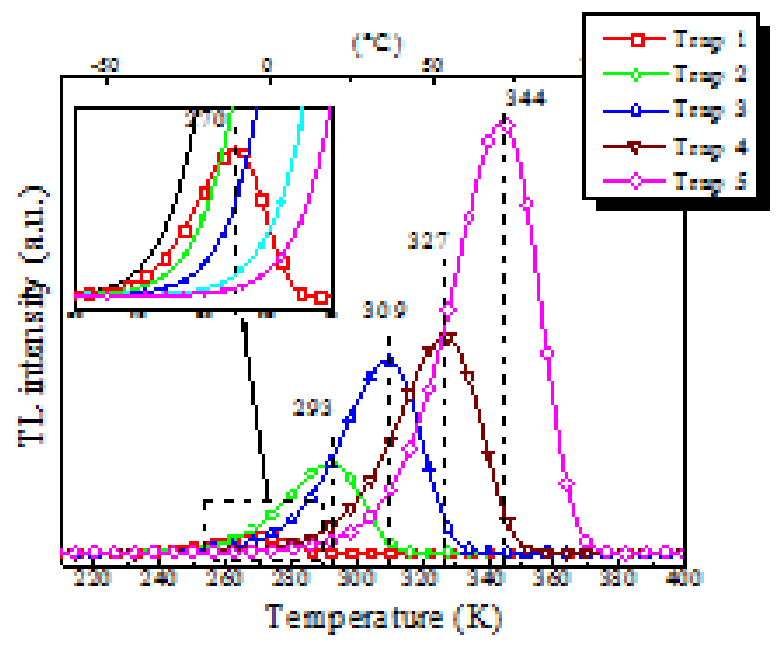

Figure 6 


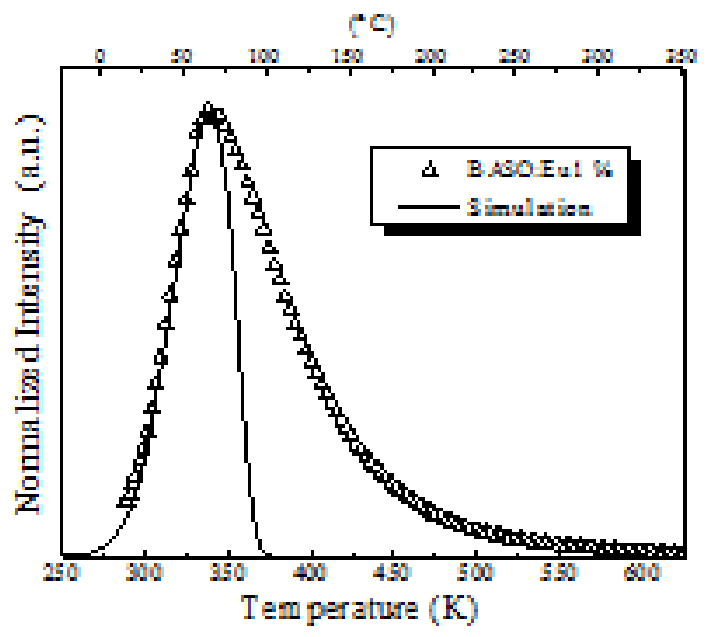

Figure 7 